\title{
Diagnostic accuracy of ultrasound in detecting the severity of abnormally invasive placentation: a systematic review and meta-analysis
}

\author{
GIORGIO PAGANI ${ }^{1}$ (D), GIUSEPPE CALI ${ }^{2}$, GANESH ACHARYA ${ }^{3,4}$, ILAN-TIMOR TRISCH ${ }^{5}$, \\ JOSE PALACIOS-JARAQUEMADA ${ }^{6}$, ALESSANDRA FAMILIARI ${ }^{7}$ (D), DANILO BUCA ${ }^{8}$, \\ LAMBERTO MANZOLI ${ }^{9}$, MARIA E. FLACCO ${ }^{10}$, FRANCESCO FANFANI ${ }^{8}$ (ID, MARCO LIBERATI ${ }^{8}$, \\ GIOVANNI SCAMBIA ${ }^{7}$ \& FRANCESCO D'ANTONIO ${ }^{4,11}$ \\ ${ }^{1}$ Department of Obstetrics and Gynecology, Fondazione Poliambulanza, Brescia, ${ }^{2}$ Department of Obstetrics and Gynecol- \\ ogy, Arnas Civico Hospital, Palermo, Italy, ${ }^{3}$ Department of Clinical Science, Intervention and Technology, Karolinska Insti- \\ tute, Stockholm, Sweden, ${ }^{4}$ Women's Health and Perinatology Research Group, Department of Clinical Medicine, Faculty \\ of Health Sciences, UiT-The Arctic University of Norway, Tromsø, Norway, ${ }^{5}$ Department of Obstetrics and Gynecology, \\ Division of Maternal-Fetal Medicine, New York University SOM, New York, NY, USA, ${ }^{6}$ Center for Medical Education and \\ Clinical Research (CEMIC), University Hospital, Buenos Aires, Argentina, ${ }^{7}$ Department of Obstetrics and Gynecology, \\ Catholic University of The Sacred Heart, Rome, ${ }^{8}$ Department of Obstetrics and Gynecology, University of Chieti, Chieti, \\ ${ }^{9}$ Department of Medical Sciences, University of Ferrara, Ferrara, ${ }^{10}$ Local Health Unit of Pescara, Pescara, Italy, and \\ ${ }^{11}$ Department of Obstetrics and Gynecology, University Hospital of Northern Norway, Tromsø, Norway
}

\section{Key words}

Abnormally invasive placenta, placenta accreta, placenta increta, placenta percreta, prenatal diagnosis, ultrasound

\section{Correspondence}

Francesco D'Antonio, Department of Clinical Medicine, Faculty of Health Sciences, UiT The Arctic University of Norway, Hansine Hansens veg 18, 9019 Tromsø, Norway. E-mail: francesco.dantonio@uit.no

\section{Conflict of interest}

The authors have stated explicitly that there are no conflicts of interest in connection with this article.

Please cite this article as: Pagani G, Cali G, Acharya G, Trisch I-T, Palacios-Jaraquemada J, et al. Diagnostic accuracy of ultrasound in detecting the severity of abnormally invasive placentation: a systematic review and metaanalysis. Acta Obstet Gynecol Scand 2017; https://doi.org/10.1111/aogs.13238

Received: 14 August 2017

Accepted: 24 September 2017

DOI: 10.1111/aogs.13238

\begin{abstract}
Introduction. Our objective was to elucidate the overall diagnostic accuracy of ultrasound in detecting the severity of abnormally invasive placentation (AIP). Material and methods. Medline, Embase, CINAHL and The Cochrane databases were searched. The ultrasound signs explored were: loss of hypoechoic (clear) zone in the placental-uterine interface, placental lacunae, bladder wall interruption, myometrial thinning, focal exophitic mass, placental lacunar flow, subplacental vascularity, and uterovesical hypervascularity. Results. Twenty studies (3209 pregnancies) were included. Ultrasound had an overall good diagnostic accuracy in identifying the depth of placental invasion with sensitivities of $90.6 \%, 93.0 \%, 89.5 \%$, and $81.2 \%$ for placenta accreta, increta, accreta/increta, and percreta, respectively; the corresponding specificities were $97.1 \%, 98.4 \%, 94.7 \%$, and $98.9 \%$. Placental lacunae had sensitivities of $74.8 \%$, $88.6 \%$, and $76.3 \%$ for the detection of placenta accreta, increta, and percreta, respectively. Sensitivity and specificity of loss of the clear zone in identifying placenta accreta were $74.9 \%$ and $92.0 \%$, whereas the corresponding figures for placenta increta and percreta were $91.6 \%$ and $76.9 \%$, and $88.1 \%$ and $71.1 \%$. Lacunar flow had sensitivities of $81.2 \%, 84.3 \%$, and $45.2 \%$ for the detection of placenta accreta, increta, and percreta respectively; the corresponding figures for specificity were $84.0 \%, 79.7 \%$, and $75.3 \%$. Sensitivity of uterovesical hypervascularity was low for the detection of placenta accreta $(12.3 \%)$ but high for placenta increta $(94.4 \%)$ and percreta $(86.2 \%)$; the corresponding figures for specificity were $90.8 \%, \quad 88.0 \%$ and $88.2 \%$, respectively. Conclusions. Ultrasound has an overall good diagnostic accuracy in recognizing the depth and the topography of placental invasion.
\end{abstract}

Abbreviations: AIP, abnormally invasive placenta; CS, cesarean section; DOR, diagnostic odds ratio; HSROC, hierarchical summary receiver characteristics curve; OR, odds ratio. 


\section{Introduction}

Abnormally invasive placentation (AIP) encompasses a spectrum of conditions characterized by an abnormal adherence and invasion of the placenta to the implantation site (1-3). Accurate prenatal diagnosis of AIP is fundamental because it has been shown to reduce the burden of maternal and fetal morbidity associated with this condition, such as severe hemorrhage, need for blood transfusion, peripartum hysterectomy and intraoperative and postoperative complications, especially by allowing implementation of preplanned management strategies (4).

Prenatal diagnosis of AIP is commonly accomplished by ultrasound during the second and third trimesters of pregnancy and has been shown to have an overall good diagnostic accuracy in women at risk, such as those with placenta previa and previous cesarean section (CS), especially when a combination of maternal characteristics and imaging signs are integrated into an individualized diagnostic algorithm (5-8).

Intra- and post-surgical outcomes of women affected by AIP are directly related to the depth and topography of placental invasion with cases affected by placenta percreta and/or showing parametrial invasion being at the highest risk of morbidity $(1,2)$. However, the actual performance of ultrasound in detecting the severity of placental uterine invasion remains elusive. Furthermore, although several ultrasound signs have been reported to be associated with AIP, the strength of association and the predictive accuracy of each ultrasound sign suggestive of AIP and the severity of placental invasion still remain unclear.

We have previously shown that ultrasound has an overall good diagnostic accuracy in detecting AIP (7). The aim of this systematic review was to elucidate the overall diagnostic accuracy of prenatal ultrasound in detecting the severity of placental invasion in women at risk; the secondary aim was to explore the strength of association and the predictive accuracy of each ultrasound sign suggestive of AIP in identifying the type of placental invasion.

\section{Material and methods}

This review was performed according to an a priori designed protocol recommended for systematic reviews and meta-analysis (9-11). Medline, Embase, CINAHL, and The Cochrane Library including The Cochrane Database of Systematic Reviews (CDSR), Database of Abstracts of Reviews of Effects (DARE), and The Cochrane Central Register of Controlled Trials (CENTRAL) were searched electronically on 23 February 2017 using combinations of the relevant medical subject heading $(\mathrm{MeSH})$ terms, key words, and word variants for "Abnormal invasive placenta" "morbidly adherent placenta" and "ultrasound" (see Supplementary material, Table S1). The search and selection criteria were restricted to English language. Reference lists of relevant articles and reviews were hand searched for additional reports. Prisma and STARD guidelines were followed $(12,13)$. The study was registered with the PROSPERO database (Registration number: CRD42017069636).

The primary outcome measure of the present systematic review was the diagnostic accuracy of ultrasound in recognizing the severity of AIP, defined by the depth and topography of placental invasion. For the depth of placental invasion, the reference standard was histopathological examination of the removed uterus (14). Placenta accreta was diagnosed when anchoring placental villi were attached to myometrium rather than decidua, but without completely invading it; placenta increta was diagnosed when chorionic villi penetrate the myometrium, and placenta percreta when chorionic villi penetrated through the myometrium to the uterine serosa or adjacent organs (14). For the assessment of the topography of placental invasion, we adopted the anatomical classification of AIP proposed by Palacios-Jaraquemada et al. (15). According to such classification, anterior placental invasion is divided into two sectors delimited by a plane perpendicular to the upper bladder axis, and the uterine sector bordering; the upper posterior bladder wall is called S1, and the uterine sector adjacent to the lower posterior wall is called S2 (Figure 1). From an anatomical perspective, $\mathrm{S} 1$ invasion refers to an invasion situated in the uterine body while S2 described invasion that is mainly located in the lower uterine segment or below it. Reference standard was the topography of invasion observed at surgery (15).

The secondary outcomes investigated were the strength of association between each ultrasound sign of AIP and the depth of placental uterine invasion and their individual predictive accuracy in detecting such invasion.

The ultrasound signs explored in the present systematic review were differentiated into those identified on gray scale and those on color Doppler ultrasound (16).

Gray-scale ultrasound signs of AIP were:

\section{Key Message}

Ultrasound has an overall good diagnostic accuracy in recognizing the depth and the topography of placental invasion. 


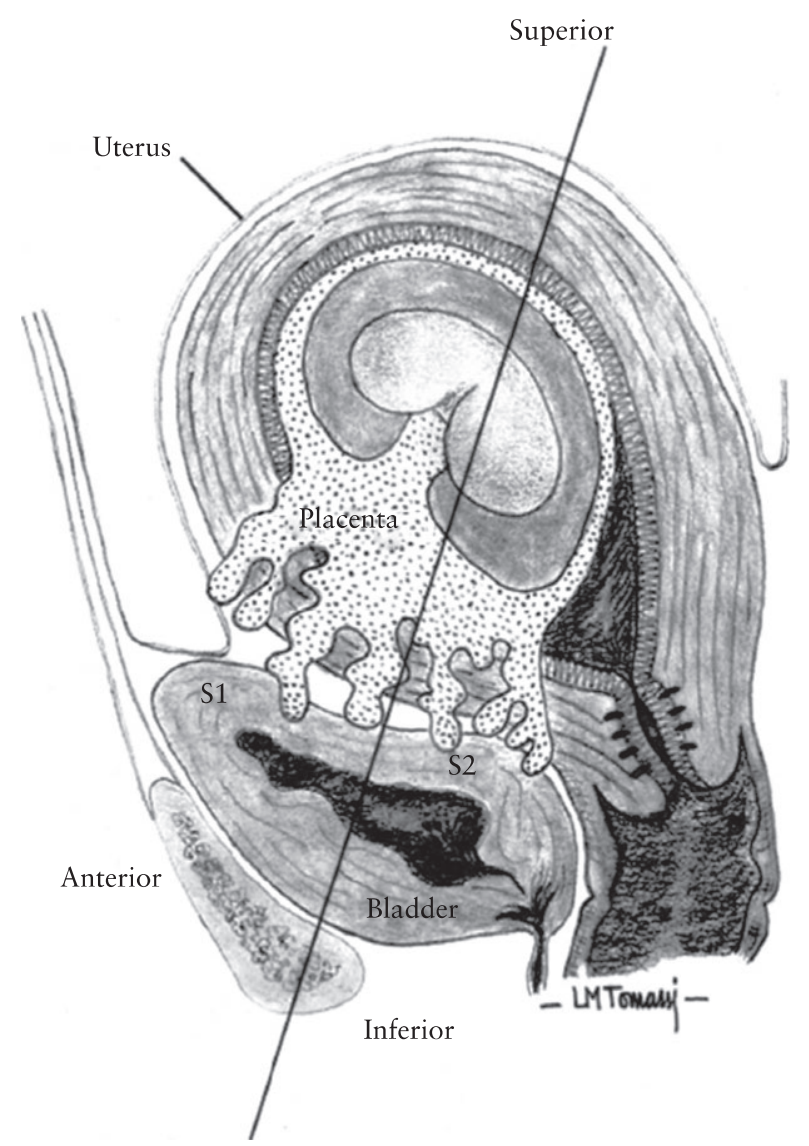

Figure 1. Anatomical representation of topographic classification of morbidly adherent placenta. Anterior placental invasion is divided into two sectors, delimited by a plane perpendicular to upper bladder axis. Upper posterior bladder wall is labeled as $\mathbf{S 1}$ and uterine sector adjacent to lower posterior wall as $\mathbf{S} 2$.

1. Loss of clear zone, defined as a loss, or irregularity, of hypoechoic plane in myometrium underneath placental bed ('clear zone')

2. Placental lacunae, defined as the presence of numerous lacunae, often containing turbulent flow visible on gray-scale imaging

3. Bladder wall interruption, defined as loss or interruption of bright bladder wall (hyperechoic band or 'line' between uterine serosa and bladder lumen)

4. Myometrial thinning, defined as thinning of myometrium overlying placenta to $<1 \mathrm{~mm}$ or undetectable

5. Focal exophytic mass, defined as placental tissue seen breaking through uterine serosa and extending beyond it; most often seen inside filled urinary bladder.

Color Doppler signs of AIP were:

1. Placental lacunar flow, defined as the presence of color Doppler signal within placental lacunae. Furthermore, lacunar flow was divided into diffuse and focal lacunar flow patterns. Diffuse lacunar flow was defined as a pattern exhibiting diffusely dilated vascular channels scattered throughout the whole placenta and the surrounding myometrial or cervical tissues, while focal lacunar flow was defined as a color Doppler pattern showing irregular sonolucent vascular lakes with turbulent lacunar flow distributed regionally or focally within the intraparenchymal placental area.

2. Subplacental vascularity, defined as striking amount of color Doppler signal seen behind the placental bed.

3. Uterovesical hypervascularity, defined as striking amount of color Doppler signal seen between myometrium and posterior wall of bladder, including vessels appearing to extend from placenta, across myometrium and beyond serosa into bladder or other organs; often running perpendicular to myometrium.

Studies were assessed according to the following criteria: population, prenatal diagnosis of AIP on ultrasound, depth of placental invasion, and study design. Only studies reporting the diagnostic accuracy of ultrasound and different ultrasound signs in detecting the severity of placental invasion based on histopathological diagnosis were considered eligible for inclusion in the present systematic review. Studies reporting exclusively a clinical diagnosis of AIP, such as difficulty or delay in placental detachment after delivery and those not differentiating among the severity of invasion were excluded. Studies reporting exclusively the prenatal diagnosis of AIP after first-trimester or second-trimester abortion as well as those reporting the prevalence of a given ultrasound sign only in cases affected by AIP were also excluded. Prospective and retrospective cohorts, case-control studies, and case series were analyzed. Opinions and studies carried out only in the second and/or third trimester of pregnancy were excluded. Case reports were also excluded in order to avoid publication bias. Studies published before 2000 were excluded, as we considered that advances in prenatal imaging techniques and improvements in the diagnosis and definition of AIP make these less relevant.

Two reviewers (FDA, GP) independently extracted data. Inconsistencies were discussed among the reviewers and consensus was reached. For those articles in which targeted information was not reported but the methodology was such that the information might have been recorded initially, the authors were contacted requesting the data. Histopathological findings and/or surgical notes were used as a reference standard. Quality of studies was assessed using the revised tool for the quality assessment of diagnostic accuracy studies (QUADAS-2) (17). Each item was scored a "yes", "no", or "unclear" if there was insufficient information to make an accurate judgment (17). 
We evaluated the predictive accuracy of prenatal ultrasound for the detection of four degrees of placental invasion: placenta accreta, increta, percreta, and a combination of placenta accreta and increta. We evaluated the association between each of the above four outcomes and: (i) an abnormal overall prenatal ultrasound index; (ii) each of the 10 prenatal ultrasound signs included in the index: placental lacunae; loss of the clear zone; myometrial thinning; exophytic mass in the bladder; bladder wall interruption; overall placental lacunar flow; diffuse placental lacunar flow; focal placental lacunar flow; subplacental vascularity; and uterovesical hypervascularity. We therefore performed $11^{*} 4=44$ separate meta-analyses, the unit of which were single comparisons of subjects with an abnormal (exposed group) vs. normal (unexposed group) ultrasound sign to predict each of the four outcomes.

For each meta-analysis, we first computed summary estimates of sensitivity, specificity, positive and negative likelihood ratios (LR+ and LR-), and diagnostic odds ratio (DOR) using the hierarchical summary receiveroperating characteristics (HSROC) model (18-20). Rutter and Gatsonis HSROC parameterization was used because it models functions of sensitivity and specificity to define a summary ROC curve, and its hierarchical modeling strategy can be used for comparisons of test accuracy when there is variability in threshold between studies (18-20). However, when the number of studies is small, the uncertainty associated with the estimation of the shape parameter could be very high, and models may fail to converge. Hence, for all meta-analyses in which less than four study estimates could be pooled, the DerSimonian-Laird random-effect model was used.

Some of the meta-analyses included observational casecontrol studies reporting zero events in one or both the compared groups, and unbalanced exposed and unexposed groups. In these cases, the best performing methods are the Mantel-Haenszel odds ratio without zero-cell continuity corrections, logistic regression and an exact method (21-23). Mantel-Haenszel odds ratios cannot be computed in studies reporting zero events in both groups, but their exclusion may cause a relevant loss of information and the potential inflation of the magnitude of the pooled exposure effect (21-23). Therefore, to keep all studies in the analyses, we performed all such meta-analyses using individual data random-effect logistic regression, with single study as the cluster unit. The pooled data sets with individual data were reconstructed using published $2 \times 2$ tables. In one meta-analysis, one of the overall pooled arms showed no events, and we had to use exact logistic regression. If a meta-analysis included only one study in the comparison, the related odds ratio was computed from the raw data of the single study.
We finally performed meta-analyses of proportions to estimate the pooled rates of placenta accreta, increta, percreta, and accreta+increta of women with and without each of the 10 ultrasound signs, respectively. Proportion metaanalyses were performed using a random-effect model to account for the inter-study heterogeneity. When only one study was available, no proportion meta-analysis was carried out. Tests for funnel plot asymmetry were not used when the total number of publications included for each outcome was $<10$. In this case, the power of the tests was too low to distinguish chance from real asymmetry. STATA command metandi (Stata Corp. College Station, TX: 2013) and Meta-Disc 1.4 (Unit of Clinical Biostatistic team of the Ramón y Cajal Hospital of Madrid, Madrid, Spain) were used to analyze the data. $(24,25)$.

\section{Results}

A total of 1018 articles were identified. After screening the abstracts, 107 full text articles were assessed with respect to their eligibility for inclusion (see Supplementary material, Table S2) and 20 studies were included in the systematic review (Table 1, Figure 2) (26-45). These 20 studies included 3209 pregnancies at risk for AIP, mainly because of the presence of placenta previa and previous CS or uterine surgery. Out of these 407 (12.7\%, 95\% CI 11.6-13.9) had AIP. The occurrences of placenta accreta, increta and percreta were $37.8 \%$ (95\% CI 33.142.7 ), $32.2 \%$ (95\% CI 27.7-37.0), and 30.0\% (95\% CI 25.6-34.7), respectively. General characteristics of the studies included in the present systematic review are reported in Table 1. Most of the included studies were retrospective series, with different gestational ages at assessment and type of ultrasound signs explored (Table 1). Quality assessment based on QUADAS-2 guidelines is shown in Figure 3. Most of the studies were of high quality, and there was a low risk of bias and low concern regarding the applicability of the studies.

Seven studies including 721 women at risk for AIP explored the diagnostic performance of ultrasound in detecting the severity of placental invasion based on histopathology. Ultrasound had an overall good diagnostic accuracy in identifying the depth of placental invasion with sensitivities of $90.6 \%$ (95\% CI 80.7-96.5), $93.0 \%$ (95\% CI 80.9-98.5), 89.5\% (95\% CI 73.2-96.3), and 81.2\% (95\% CI 51.8-94.6) for placenta accreta, increta, accreta/increta, and percreta, respectively; the corresponding figures for specificity were 97.1\% (95\% CI 95.4-98.3), 98.4 (95\% CI 97.0-99.2), 94.7 (95\% CI 91.0-96.9), and 98.9 (95\% CI 95.0-100). Diagnostic accuracy of ultrasound in detecting the depth of placental invasion is shown in Table 2 and Figure 4. Only two studies (30,39) explored the role of ultrasound in identifying the 


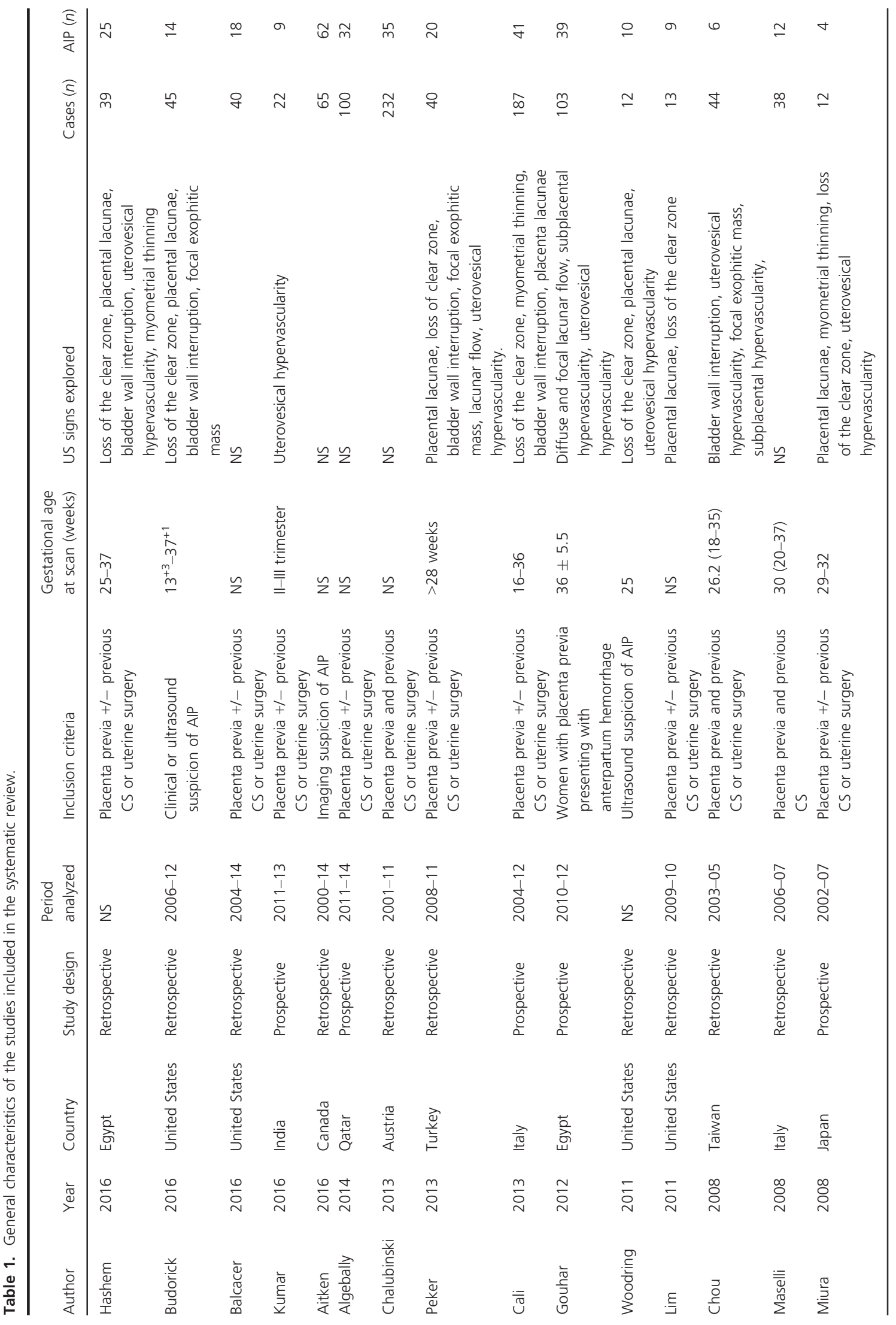




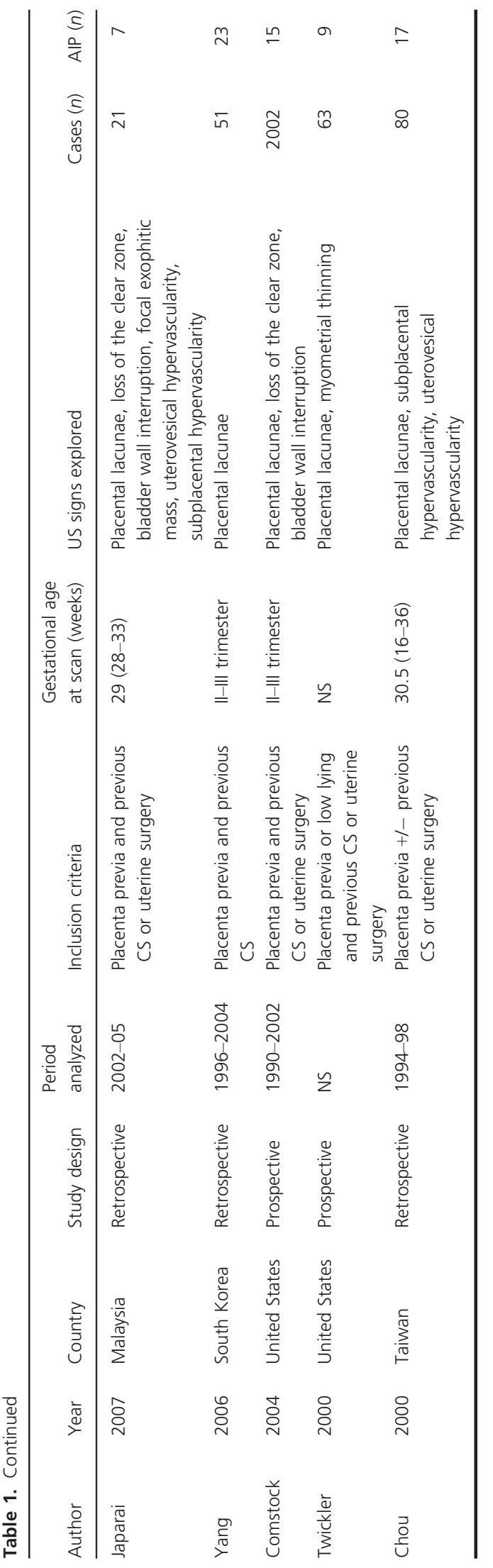

topography of the invasion. Overall, ultrasound correctly identified $93.4 \%$ (95\% CI $64.7-100)$ of women with S1 and $90.3 \%$ (95\% CI 80.7-97.4) of those with S2 invasion confirmed at surgery.

\section{Ultrasound signs of AIP}

Gray-scale ultrasound. Nine studies explored the strength of association between placental lacunae and the severity of placental invasion. The presence of lacunae was independently associated with placenta accreta, increta, and percreta (see Supplementary material, Table S3) with OR of 7.8, 16.1, and 8.2 respectively. When translating these findings into figures of diagnostic accuracy, placental lacunae had sensitivities of $74.8 \%(95 \%$ CI 55.4-87.6), 88.6\% (95\% CI 55.3-98.0), and 76.3\% (95\% CI 42.2-93.4) for the detection of placenta accreta, increta, and percreta respectively, whereas the corresponding figures for specificity were $87.9 \%$ (95\% CI $52.6-97.9$ ), 77.4\% (95\% CI $46.8-93.0$ ), and $74.0 \%$ (95\% CI 45.0 90.9). DOR of placental lacunae in the detection of the depth of placental invasion was 18.3, 27.7, and 9.2 for placenta accreta, increta, and percreta. The prevalence of the different types of AIP in women with and without placental lacunae on ultrasound is reported in the Supplementary material (Table S4).

Ten studies explored the strength of association and the predictive accuracy of the loss of the clear zone in recognizing the depth of placental invasion. Loss of the clear zone was associated with a higher risk of placenta accreta, increta, and percreta, with OR of $23.8,20.8$, and 13.0 respectively. Sensitivity and specificity of loss of the clear zone in identifying placenta accreta were $74.9 \%$ (95\% CI 33.5-94.6) and 92.0\% (95\% CI 68.8-98.3), whereas the corresponding figures for placenta increta and percreta were $91.6 \%$ (95\% CI 59.9-98.8) and 76.9\% (95\% CI 45.4-93.0), and 88.1\% (95\% CI 64.7-96.8) and 71.1\% (95\% CI 42.2-89.2) (Tables 3-6; see Supplementary material, Tables S3, S4).

Only three studies explored the association between myometrial thinning and different types of AIP. The presence of an abnormal myometrial thickness, defined as $<1 \mathrm{~mm}$, was associated with a higher risk of every type of AIP. Sensitivity and specificity were $100 \%$ (95\% CI 31.0 $100)$ and $85.0 \%$ (95\% CI 72.9-92.5) for placenta accreta, $100 \%$ (95\% CI $47.8-100)$ and $74.3 \%$ (95\% CI 62.4-84.0) for placenta increta and $85.7 \%$ (95\% CI 57.2-98.2) and 76.0\% (95\% CI 66.4-84.0) for placenta percreta (Tables 3-6).

The presence of a focal exophitic mass extending into the bladder was analyzed only for cases with placenta percreta and was explored only by one study, thus precluding a pooled data synthesis. Focal exophitic mass had sensitivity of $16.7 \%$ (95\% CI $0.42-64.2$ ), a specificity of 

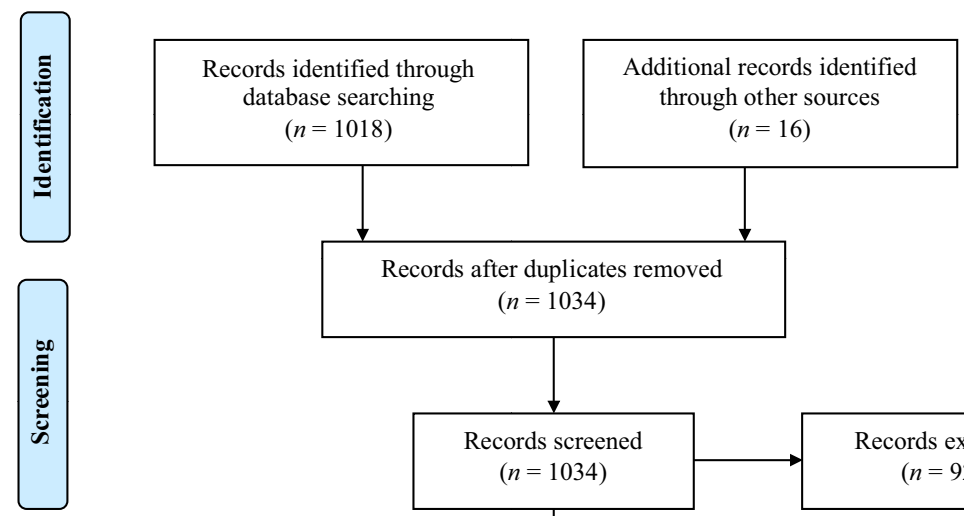

Records after duplicates removed $(n=1034)$
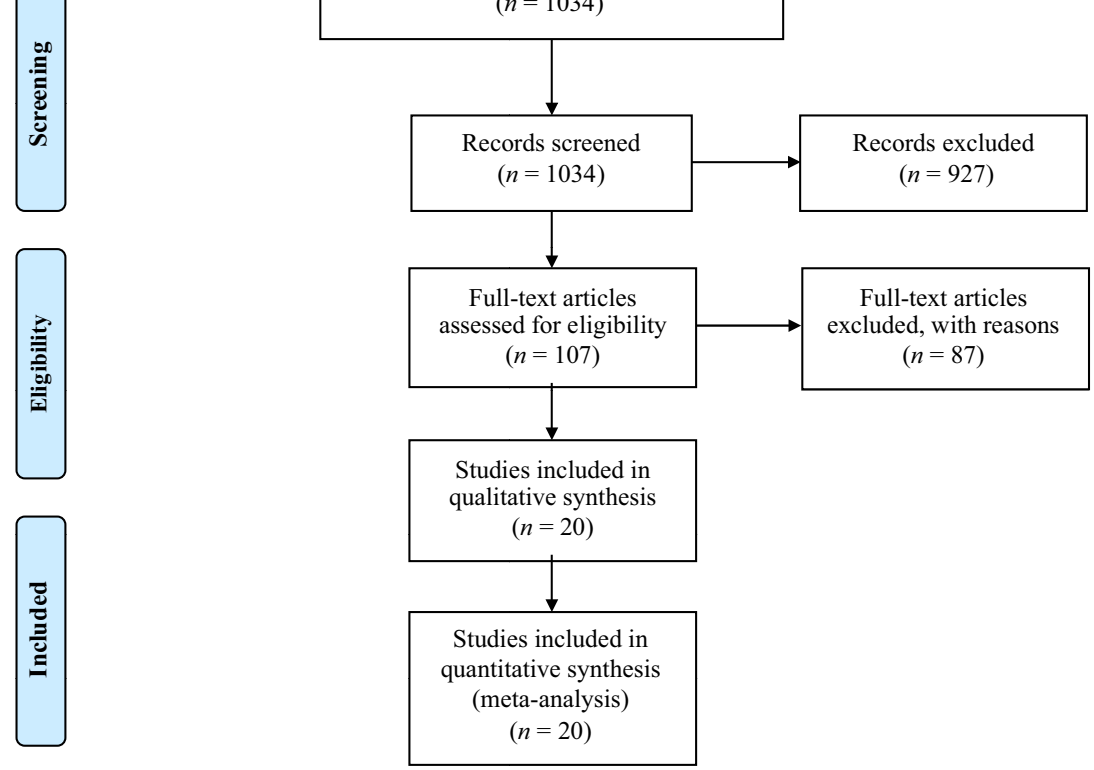

Figure 2. Systematic review flowchart. [Color figure can be viewed at wileyonlinelibrary.com].

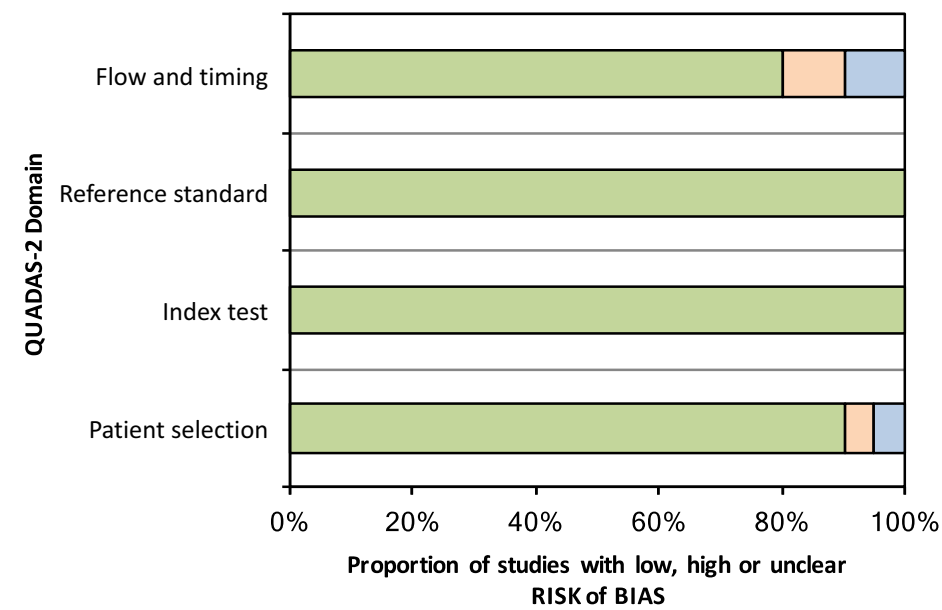

口Low $\square$ High DUnclear

Figure 3. QUADAS-2 assessment of the studies included in the systematic review. [Color figure can be viewed at wileyonlinelibrary.com].

$100 \%$ (95\% CI $88.6-100)$, and a DOR of 21.0 (95\% CI $0.76-583)$ in detecting placenta percreta (41).

Finally, seven studies explored the strength of association and predictive accuracy of bladder wall abnormalities in identifying the severity of AIP (see Supplementary material, Tables S3, S4). The presence of any abnormality at the bladder-uterine interface, such as loss or interruption of bright bladder wall, was significantly associated with placenta accreta, increta, and percreta, with OR of 5.3, 27.6, and 77.6, respectively. Abnormalities of the bladder wall had a sensitivity, a specificity, and a DOR of 17.0\% (95\% CI 0.06-85.8), 96.8\% (95\% CI 86.0-99.3), and $6.13(0.13-294.1)$ in identifying cases with placenta accreta. The diagnostic performance progressively increased in terms of sensitivity for the detection of placenta increta $(46.1 \%, 95 \%$ CI $11.0-85.5)$ and percreta 
Table 2. Summary estimates of sensitivity, specificity, positive and negative likelihood ratios (LR+ and LR-) and diagnostic odds ratio (DOR) of ultrasound to detect each degree of placental invasion.

\begin{tabular}{lclllll}
\hline & $\begin{array}{l}\text { No.of studies } \\
\text { (sample) }\end{array}$ & $\begin{array}{l}\text { Sensitivity } \\
\%(95 \% \mathrm{Cl})\end{array}$ & $\begin{array}{l}\text { Specificity } \\
\%(95 \% \mathrm{Cl})\end{array}$ & DOR (95\% Cl) & LR+ (95\% Cl) & LR- (95\% Cl) \\
\hline Placenta accreta & $6(681)$ & $90.6(80.7-96.5)$ & $97.1(95.4-98.3)$ & $193(72.5-514)$ & $23.6(14.9-34.3)$ & $0.15(0.08-0.30)$ \\
Placenta increta & $5(660)$ & $93.0(80.9-98.5)$ & $98.4(97.0-99.2)$ & $503(137-1846)$ & $37.5(19.2-73.4)$ & $0.14(0.04-0.49)$ \\
Placenta accreta/increta & $7(721)$ & $89.5(73.2-96.3)$ & $94.7(91.0-96.9)$ & $151(38.9-582)$ & $16.8(9.48-29.6)$ & $0.11(0.04-0.31)$ \\
Placenta percreta & $7(721)$ & $81.2(51.8-94.6)$ & $98.9(95.0-100)$ & $399(54.5-2928)$ & $75.8(16.0-359)$ & $0.19(0.06-0.59)$ \\
\hline
\end{tabular}

Computations were based upon hierarchical summary receiver operating characteristic (HSROC) model.

(62.0\%, 95\% CI 23.2-89.8); the corresponding figures for specificity were $97.3 \%(95 \%$ CI $91.0-99.3)$ and $97.5 \%$ (95\% CI 90.9-99.4).

Color Doppler ultrasound. Five studies explored the diagnostic performance of lacunar flow in identifying the severity of placental invasion. The presence of lacunar flow in women at risk of AIP was significantly associated with the occurrence of placenta accreta (OR 21.6), increta (OR 20.4), and percreta (OR 2.51). Lacunar flow had sensitivities of $81.2 \%$ (95\% CI $57.2-93.3), 84.3 \%$ (95\% CI 50.8-96.5), and 45.2\% (95\% CI 27.3-64.0) for the detection of placenta accreta, increta, and percreta respectively; the corresponding figures for specificity were $84.0 \%(95 \%$ CI 65.4-93.6), 79.7\% (95\% CI 57.4-91.9), and 75.3\% (95\% CI 69.8-80.2). Diagnostic accuracy of diffuse and overall lacunar flow in identifying the severity of AIP is shown in Tables 3-6.

Three studies explored the diagnostic accuracy of subplacental hypervascularity, defined as striking amount of color Doppler signal seen in placental bed. Subplacental hypervascularity had a low sensitivity in detecting placenta accreta $(40.7 \%, 95 \%$ CI 22.4-61.2), increta $(17.4 \%, 95 \%$ CI 5.0-38.8), and percreta (40.0\%, 95\% CI 12.2-73.8), while specificity was 95.5\% (95\% CI 91.3-98.0), 93.8\% (95\% CI 88.8-97.0), and 92.5\% (95\% CI 85.1-96.9), respectively.

Finally, seven studies explored the predictive accuracy of uterovesical hypervascularity in detecting the severity of AIP. Uterovesical hypervascularity was significantly associated with placenta accreta (OR 4.6), increta (OR 32.4) and percreta (OR 48.9). Sensitivity was low for the detection of placenta accreta $(12.3 \%, 95 \%$ CI $2.59-100)$ but high for placenta increta $(94.4 \%, 95 \%$ CI 29.2-100) and percreta $(86.2 \%, 95 \%$ CI $60.0-96.3)$; the corresponding figures for specificity were $90.8 \%$ (95\% CI 75.2-97.0), $88.0 \%$ (95\% CI 72.8-95.3), and $88.2 \%$ (95\% CI 71.9 95.6), respectively.

\section{Discussion}

The findings from this systematic review showed that ultrasound has an overall good diagnostic accuracy in recognizing the depth and the topography of placental invasion. Among the different ultrasound signs of AIP, myometrial thinning, bladder wall interruption, and uterovesical hypervascularity were those associated with the most severe types of AIP, such as placenta percreta, and showed an overall good predictive accuracy.

The small number of included studies, their retrospective design, heterogeneity in ultrasound signs explored, and gestational ages at assessment represent the main limitations of the present systematic review. Furthermore, the large majority of studies exploring the predictive accuracy of ultrasound in detecting AIP did not report the diagnostic performance of ultrasound in detecting the topography of placental invasion according to the classification system provided by Palacios-Jaraquemada et al. (15). Knowledge of the exact topography of placental invasion is fundamental in planning the management of AIP, especially when a procedure such as hysterectomy is planned. Assessment of the topography of placental invasion has been initially reported using fetal MRI and only recently using ultrasound, which explains the very small number of studies exploring this parameter.

Gestational age at assessment is another important issue. Although the majority of included cases were assessed in the third trimester of pregnancy, lack of information of the precise gestational age at scan precluded stratification of the analysis according to the time at assessment. This is fundamental, because AIP is a progressive condition and it may be entirely possible that the ultrasound appearance of the different ultrasound signs of AIP can change/evolve though gestation.

The risk of AIP is directly related to the number of previous CS and models integrating pregnancy characteristics and ultrasound signs have been shown to predict AIP more accurately than imaging alone, although such studies did not explore the performance of these models in identifying the severity of placental invasion (8). In the present systematic review, we could not stratify the analysis according to the number of previous CS or other maternal characteristics, in view of the lack of individual data. 


\section{Placenta accreta}

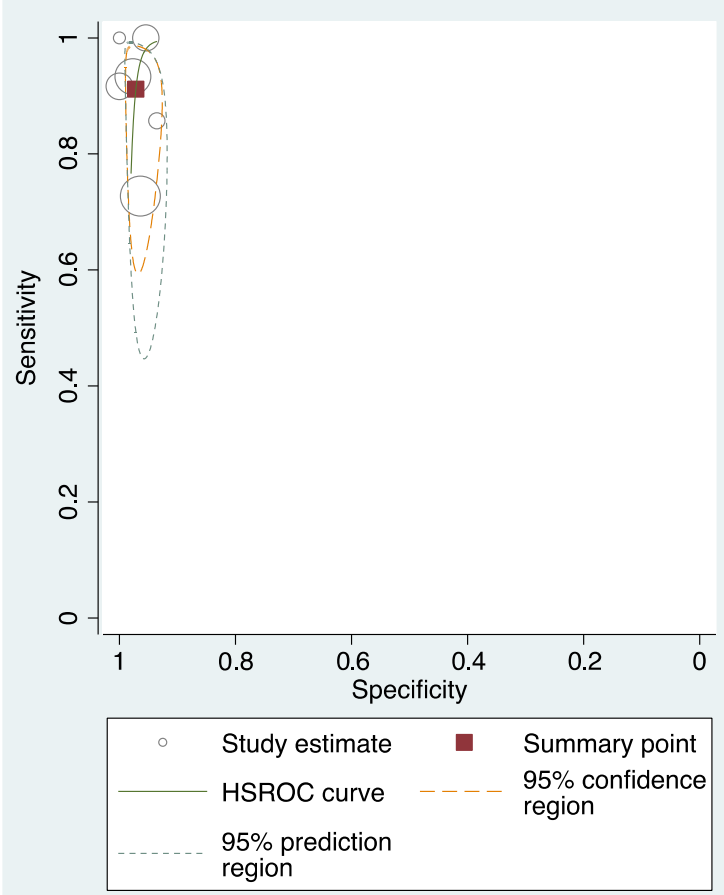

Placenta accreta/increta

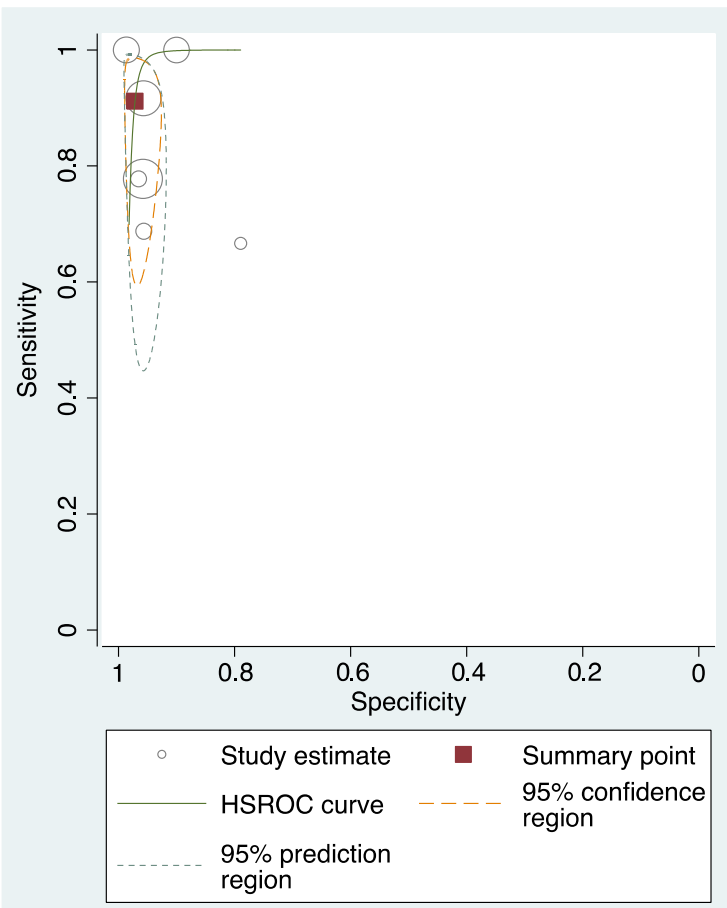

\section{Placenta increta}

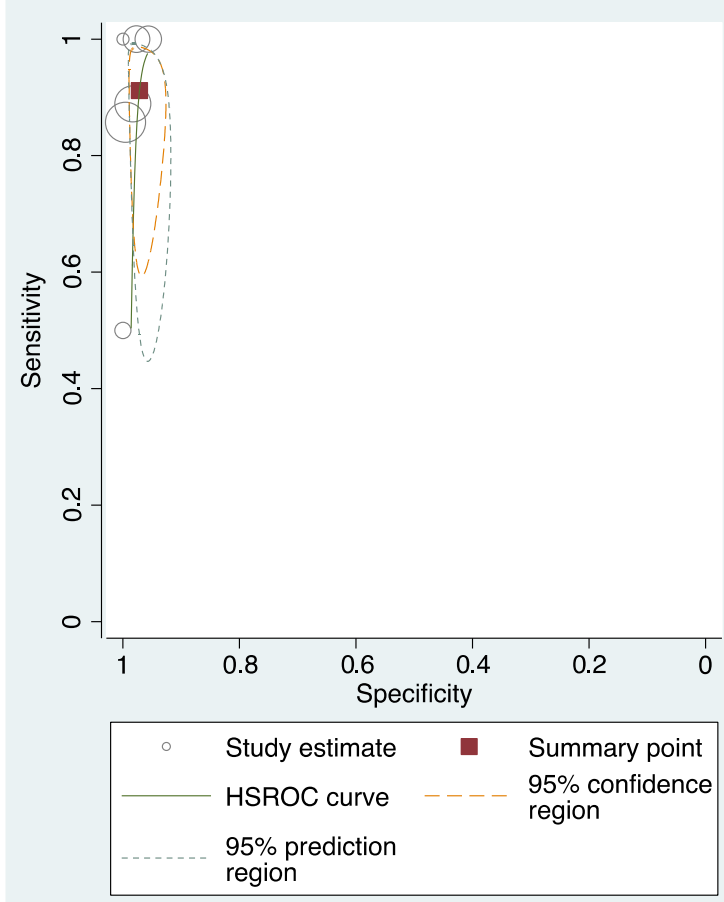

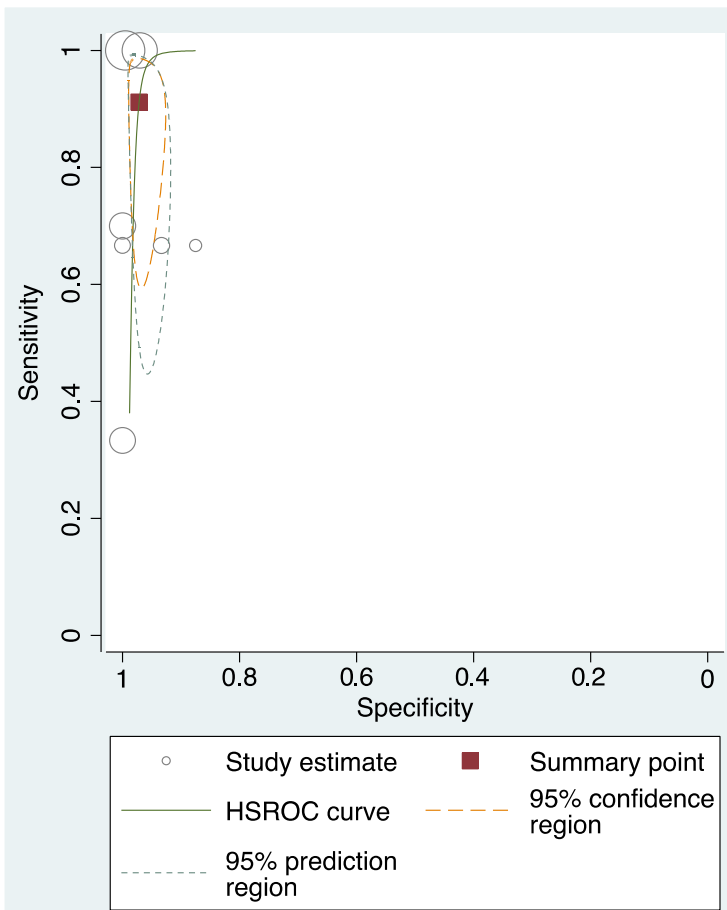

Figure 4. Hierarchical receiver operating characteristics (HSROC) curve of the predictive accuracy of prenatal ultrasound in detecting the depth of placental invasion. [Color figure can be viewed at wileyonlinelibrary.com]. 
Table 3. Summary estimates of sensitivity, specificity, positive and negative likelihood ratios (LR+ and LR-) and diagnostic odds ratio (DOR) of each ultrasound sign to predict a diagnosis of placenta accreta.

\begin{tabular}{lcccccc}
\hline Ultrasound sign & $\begin{array}{l}\text { No. of } \\
\text { studies }\end{array}$ & $\begin{array}{l}\text { Sensitivity } \\
\%(95 \% \mathrm{Cl})\end{array}$ & $\begin{array}{l}\text { Specificity } \\
\%(95 \% \mathrm{Cl})\end{array}$ & DOR (95\% CI) & LR+ $(95 \% \mathrm{Cl})$ & $\mathrm{LR}-(95 \% \mathrm{Cl})$ \\
\hline 1. Placental lacunae & $7^{\dagger}$ & $74.8(55.4-87.6)$ & $87.9(52.6-97.9)$ & $18.3(2.02-165.8)$ & $5.37(1.19-24.15)$ & $0.29(0.13-0.65)$ \\
2. Loss of clear zone & $8^{\dagger}$ & $74.9(33.5-94.6)$ & $92.0(688-98.3)$ & $34.1(2.22-254.8)$ & $9.33(1.72-50.70)$ & $0.27(0.07-1.08)$ \\
3. Myometrial thinning & $1^{*}$ & $100(31.0-100)$ & $85.0(72.9-92.5)$ & $37.9(1.81-796)$ & $6.61(3.65-12.2)$ & - \\
4. Focal exophitic mass & - & - & - & - & - & - \\
5. Bladder wall interruption & $6^{\dagger}$ & $17.0(0.06-85.8)$ & $96.8(86.0-99.3)$ & $6.13(0.13-294.1)$ & $5.26(0.19-145.9)$ & $0.86(0.48-1.54)$ \\
6. Lacunar flow (overall) & $5^{\dagger}$ & $81.2(57.2-93.3)$ & $84.0(65.4-93.6)$ & $22.8(8.35-62.2)$ & $5.09(2.40-10.8)$ & $0.22(0.09-0.53)$ \\
7. Diffuse lacunar flow & $3^{*}$ & $33.3(16.5-54.0)$ & $91.5(86.4-95.2)$ & $5.29(0.82-34.2)$ & $3.32(0.86-12.8)$ & $0.72(0.38-1.36)$ \\
8. Focal lacunar flow & $2^{*}$ & $65.0(40.8-84.6)$ & $90.2(84.5-90.3)$ & $21.0(2.70-163)$ & $7.93(1.85-33.9)$ & $0.42(0.23-0.75)$ \\
9. Subplacental vascularity & $3^{*}$ & $40.7(22.4-61.2)$ & $95.5(91.3-98.0)$ & $11.9(3.30-43.1)$ & $7.01(2.50-19.6)$ & $0.68(0.49-0.96)$ \\
10. Uterovesical hypervascularity & $4^{\dagger}$ & $12.3(2.59-100)$ & $90.8(75.2-97.0)$ & $4.99(0.25-101)$ & $2.53(0.33-19.3)$ & $0.65(0.29-1.50)$ \\
\hline
\end{tabular}

Depending on the number of studies, computations were based upon DerSimonian-Laird random-effect $(*)$ or hierarchical summary receiver operating characteristic (HSROC) model $\left({ }^{\dagger}\right)$.

Table 4. Summary estimates of sensitivity, specificity, positive and negative likelihood ratios (LR+ and LR-) and diagnostic odds ratio (DOR) of each ultrasound sign to predict a diagnosis of placenta increta.

\begin{tabular}{lcccccc}
\hline Ultrasound sign & $\begin{array}{l}\text { No. of } \\
\text { studies }\end{array}$ & $\begin{array}{l}\text { Sensitivity } \\
\%(95 \% \mathrm{Cl})\end{array}$ & $\begin{array}{l}\text { Specificity } \\
\%(95 \% \mathrm{Cl})\end{array}$ & DOR (95\% CI) & LR+ $(95 \% \mathrm{Cl})$ & $\mathrm{LR}-(95 \% \mathrm{Cl})$ \\
\hline 1. Placental lacunae & $7^{\dagger}$ & $88.6(55.3-98.0)$ & $77.4(46.8-93.0)$ & $26.7(2.76-257.8)$ & $3.92(1.35-11.39)$ & $0.15(0.03-0.77)$ \\
2. Loss of clear zone & $8^{\dagger}$ & $91.6(59.9-98.8)$ & $76.9(45.4-93.0)$ & $36.3(6.26-210.7)$ & $3.97(1.47-10.7)$ & $0.11(0.02-0.59)$ \\
3. Myometrial thinning & $2^{*}$ & $100(47.8-100)$ & $74.3(62.4-84.0)$ & $6.04(0.32-112)$ & $2.19(0.46-10.2)$ & $0.36(0.05-2.51)$ \\
4. Focal exophitic mass & - & - & - & - & - & - \\
5. Bladder wall interruption & $6^{\dagger}$ & $46.1(11.0-85.5)$ & $97.3(91.0-99.3)$ & $31.3(8.09-120.9)$ & $17.3(7.13-42.1)$ & $0.55(0.23-1.32)$ \\
6. Lacunar flow (overall) & $4^{\dagger}$ & $84.3(50.8-96.5)$ & $79.7(57.4-91.9)$ & $21.0(5.28-83.8)$ & $4.15(1.98-8.67)$ & $0.20(0.05-0.71)$ \\
7. Diffuse lacunar flow & $2^{*}$ & $43.5(23.2-65.5)$ & $94.0(89.6-97.4)$ & $15.6(1.82-133)$ & $10.3(1.02-105)$ & $0.61(0.43-0.87)$ \\
8. Focal lacunar flow & $2^{*}$ & $60.9(38.5-80.3)$ & $90.6(85.0-94.7)$ & $14.2(5.19-38.8)$ & $6.18(3.40-11.2)$ & $0.44(0.27-0.74)$ \\
9. Subplacental vascularity & $2^{*}$ & $17.4(5.0-38.8)$ & $93.8(88.8-97.0)$ & $2.81(0.80-9.90)$ & $2.48(0.85-7.25)$ & $0.89(0.74-1.08)$ \\
10. Uterovesical hypervascularity & $5^{\dagger}$ & $94.4(29.2-100)$ & $88.0(72.8-95.3)$ & $124(5.90-2624)$ & $7.88(3.72-16.7)$ & $0.06(0.00-1.93)$ \\
\hline
\end{tabular}

Depending on the number of studies, computations were based upon DerSimonian-Laird random effect $(*)$ or hierarchical summary receiver operating characteristic (HSROC) model $\left({ }^{\dagger}\right)$.

Finally, the number of ultrasound criteria used to diagnose the different type of AIP were not specified in the majority of the included studies.

Despite these limitations, the present systematic review represents the most comprehensive assessment of the diagnostic accuracy of ultrasound in detecting the severity of invasion in women affected by AIP.

The increased incidence of CS rate in developed countries has made AIP a relatively common anomaly, underlying the need for an accurate prenatal diagnosis. Prenatal diagnosis of AIP has been shown to significantly reduce the burden of intra- and post-surgical complications associated with this anomaly, allowing a preplanned management of the condition (4). Although ultrasound can accurately predict AIP, its diagnostic performance in recognizing the severity of invasion has still to be ascertained. Severe types of AIP, such as placenta percreta, are associated with a higher risk of adverse maternal outcomes, such as severe hemorrhage, need for transfusion, bladder injuries, and admission to intensive care unit. It is therefore desirable to identify those women at higher risk of placenta percreta so as to plan an appropriate surgical management $(1,2)$. The data from the present review showed that ultrasound has an overall good accuracy in identifying women affected by placenta percreta with a sensitivity of $81.2 \%$ (95\% CI 51.8-94.6) and a specificity of $98.9 \%$ (95\% CI 95.0-100). Despite these encouraging results, about $20 \%$ of women with placenta percreta remain undiagnosed, which underscores the need to develop more accurate prediction models for these severe types of AIP.

Outcome of women affected by AIP is not only affected by the depth but also by the extent of the invasion. Assessing the precise topography of placental invasion is challenging and has been initially reported only using fetal MRI (15). Antenatal MRI has been reported to provide an accurate anatomical description of the area invaded by the placenta (6). This evaluation is 
Table 5. Summary estimates of sensitivity, specificity, positive and negative likelihood ratios (LR+ and LR-) and diagnostic odds ratio (DOR) of each ultrasound sign to predict a diagnosis of placenta accreta+increta.

\begin{tabular}{lcccccc}
\hline Ultrasound sign & $\begin{array}{l}\text { No. of } \\
\text { studies }\end{array}$ & $\begin{array}{l}\text { Sensitivity } \\
\%(95 \% \mathrm{Cl})\end{array}$ & $\begin{array}{l}\text { Specificity } \\
\%(95 \% \mathrm{Cl})\end{array}$ & DOR (95\% Cl) & LR+ $(95 \% \mathrm{Cl})$ & LR- $(95 \% \mathrm{Cl})$ \\
\hline 1. Placental lacunae & $9^{\dagger}$ & $74.5(58.5-85.8)$ & $86.0(59.9-96.2)$ & $17.9(3.00-107.2)$ & $5.32(1.46-19.29)$ & $0.30(0.15-0.56)$ \\
2. Loss of clear zone & $10^{\dagger}$ & $79.6(51.7-93.4)$ & $86.4(64.8-95.7)$ & $24.8(5.65-108.7)$ & $5.86(2.11-16.25)$ & $0.24(0.09-0.63)$ \\
3. Myometrial thinning & $3^{*}$ & $54.2(32.8-74.4)$ & $74.4(64.2-73.1)$ & $3.73(0.21-65.1)$ & $1.89(0.51-6.96)$ & $0.51(0.06-4.42)$ \\
4. Focal exophitic mass & - & - & - & - & - & - \\
5. Bladder wall interruption & $7^{\dagger}$ & $29.8(5.13-76.8)$ & $98.4(90.7-99.8)$ & $26.8(1.60-449.2)$ & $19.13(1.78-205.31)$ & $0.71(0.39-1.32)$ \\
6. Lacunar flow (overall) & $5^{\dagger}$ & $82.0(60.6-93.1)$ & $94.4(72.6-99.1)$ & $76.9(20.5-288)$ & $14.6(2.92-73.3)$ & $0.19(0.08-0.43)$ \\
7. Diffuse lacunar flow & $3^{*}$ & $38.0(24.7-52.8)$ & $96.8(92.6-98.9)$ & $13.3(4.72-37.4)$ & $7.67(3.24-18.1)$ & $0.67(0.44-0.99)$ \\
8. Focal lacunar flow & $2^{*}$ & $62.8(46.7-67.0)$ & $98.6(94.9-99.8)$ & $79.8(19.6-325)$ & $29.8(8.56-104)$ & $0.38(0.26-0.56)$ \\
9. Subplacental vascularity & $3^{*}$ & $9.3(5.7-14.2)$ & $70.0(55.4 .8-82.1)$ & $24.8(11.0-56.1)$ & $1.22(1.02-1.44)$ & $0.34(0.20-0.61)$ \\
10. Uterovesical hypervascularity & $7^{\dagger}$ & $59.1(10.8-94.5)$ & $87.2(73.4-94.4)$ & $9.86(0.73-133)$ & $4.62(1.31-16.3)$ & $0.47(0.11-2.03)$ \\
\hline
\end{tabular}

Depending on the number of studies, computations were based upon DerSimonian-Laird random-effect $(*)$ or hierarchical summary receiver operating characteristic (HSROC) model ( $\left(^{\dagger}\right)$.

Table 6. Summary estimates of sensitivity, specificity, positive and negative likelihood ratios (LR+ and LR-) and diagnostic odds ratio (DOR) of each ultrasound sign to predict a diagnosis of placenta percreta.

\begin{tabular}{lclllll}
\hline Ultrasound sign & $\begin{array}{l}\text { No. of } \\
\text { studies }\end{array}$ & $\begin{array}{l}\text { Sensitivity } \\
\%(95 \% \mathrm{Cl})\end{array}$ & $\begin{array}{l}\text { Specificity } \\
\%(95 \% \mathrm{Cl})\end{array}$ & DOR (95\% Cl) & LR+ $(95 \% \mathrm{Cl})$ & LR- (95\% Cl) \\
\hline 1. Placental lacunae & $8^{\dagger}$ & $76.3(42.2-93.4)$ & $74.0(45.0-90.9)$ & $9.2(1.10-76.3)$ & $2.94(1.02-8.41)$ & $0.32(0.09-1.10)$ \\
2. Loss of clear zone & $9^{\dagger}$ & $88.1(64.7-96.8)$ & $71.1(42.2-89.2)$ & $18.2(3.61-92.08)$ & $3.04(1.32-7.03)$ & $0.17(0.05-0.55)$ \\
3. Myometrial thinning & $3^{*}$ & $85.7(57.2-98.2)$ & $76.0(66.4-84.0)$ & $8.99(0.90-89.5)$ & $2.81(0.88-9.01)$ & $0.33(0.09-1.16)$ \\
4. Focal exophitic mass & $1^{*}$ & $16.7(0.42-64.2)$ & $100(88.6-100)$ & $21.0(0.76-583)$ & - & $0.83(0.58-1.19)$ \\
5. Bladder wall interruption & $7^{\dagger}$ & $62.0(23.2-89.8)$ & $97.5(90.9-99.4)$ & $63.9(7.80-523.3)$ & $24.9(5.89-105.64)$ & $0.39(0.14-1.11)$ \\
6. Lacunar flow (overall) & $3^{*}$ & $45.2(27.3-64.0)$ & $75.3(69.8-80.2)$ & $2.27(0.99-5.21)$ & $1.67(1.16-2.40)$ & $0.83(0.55-1.26)$ \\
7. Diffuse lacunar flow & $1^{*}$ & $50.0(18.7-81.3)$ & $87.1(78.6-93.2)$ & $6.75(1.30-33.4)$ & $3.88(1.72-8.75)$ & $0.57(0.31-1.07)$ \\
8. Focal lacunar flow & $1^{*}$ & $20.0(2.52-55.6)$ & $80.6(71.2-88.1)$ & $1.04(0.10-5.88)$ & $1.03(0.28-3.82)$ & $0.99(0.72-1.37)$ \\
9. Subplacental vascularity & $1^{*}$ & $40.0(12.2-73.8)$ & $92.5(85.1-96.9)$ & $8.19(1.33-44.0)$ & $5.31(1.88-15.1)$ & $0.65(0.39-1.08)$ \\
10. Uterovesical hypervascularity & $6^{\dagger}$ & $86.2(60.0-96.3)$ & $88.2(71.9-95.6)$ & $46.7(8.31-263)$ & $7.31(2.82-19.0)$ & $0.16(0.05-0.53)$ \\
\hline
\end{tabular}

Depending on the number of studies, computations were based upon DerSimonian-Laird random-effect (*) or hierarchical summary receiver operating characteristic (HSROC) model (†).

usually accomplished by obtaining sagittal images and dividing the anterior placental invasion into two sectors delimited by a plane perpendicular to the upper bladder axis, and the uterine sector bordering; the upper posterior bladder wall is called S1, and the uterine sector adjacent to the lower posterior wall is called S2. The importance of this classification system relies on the fact that it can be used to tailor the optimal surgical approach. In a recent large series including more than 500 cases of AIP studied with antenatal MRI, PalaciosJaraquemada et al. (15) demonstrated that although MRI did not add any relevant information in terms of diagnosis of AIP when compared with ultrasound, it was able to clearly delineate the topography or extension of the invasion, so allowing a choice ot be made between the optimal preplanned surgical management. In the present systematic review, assessment of the topography of placental invasion was assessed only by a small proportion of the included studies; overall ultrasound could identify $93.4 \%$ and $90.3 \%$ of cases with S1 and S2 invasion, respectively. These results are encouraging and suggest that ultrasound can reliably describe the topography of placental invasion, although this requires confirmation in larger studies. However, until more robust evidence is available, we think that in women with an ultrasound diagnosis of AIP, MRI should be considered to assess the precise topography of placental invasion, especially when a resective procedure such as hysterectomy is planned.

Assessment of individual ultrasound signs of AIP should be viewed with caution. Observation of one sign is likely to increase the chance of detecting others, because the signs are not looked for in isolation. In the present systematic review, color Doppler signs were more predictive of severe types of AIP, although none of them was specific for placenta percreta.

Recently, multiparametric prediction models integrating imaging signs and pregnancy characteristics, such as 
the number of previous CS, have been shown to predict AIP more accurately than imaging alone (8). However, such models do not differentiate among the different types of AIP and cannot be used in clinical practice to estimate the risk of the most severe types of placental invasion. Further large prospective studies integrating pregnancy characteristics and imaging signs are needed to construct accurate predictive models for different types of placental invasion.

\section{Funding statement}

No funding was obtained for this systematic review.

\section{References}

1. Oyelese Y, Smulian JC. Placenta previa, placenta accreta, and vasa previa. Obstet Gynecol. 2006;107:927-41.

2. Belfort MA. Placenta accreta. Am J Obstet Gynecol. 2010;203:430-9.

3. D'Antonio F, Palacios-Jaraquemada J, Lim PS, Forlani F, Lanzone A, Timor-Tritsch I, et al. Counseling in fetal medicine: evidence-based answers to clinical questions on morbidly adherent placenta. Ultrasound Obstet Gynecol. 2016;47:290-301.

4. Tikkanen M, Paavonen J, Loukovaara M, Stefanovic V. Antenatal diagnosis of placenta accreta leads to reduced blood loss. Acta Obstet Gynecol Scand. 2011;90:1140-6.

5. Comstock $\mathrm{CH}$, Bronsteen RA. The antenatal diagnosis of placenta accreta. BJOG. 2014;121:171-82.

6. D’Antonio F, Iacovella C, Palacios-Jaraquemada J, Bruno $\mathrm{CH}$, Manzoli L, Bhide A. Prenatal identification of invasive placentation using magnetic resonance imaging: systematic review and meta-analysis. Ultrasound Obstet Gynecol. 2014;44:8-16.

7. D’Antonio F, Iacovella C, Bhide A. Prenatal identification of invasive placentation using ultrasound: systematic review and meta-analysis. Ultrasound Obstet Gynecol. 2013;42:509-17.

8. Rac MW, Dashe JS, Wells CE, Moschos E, McIntire DD, Twickler DM. Ultrasound predictors of placental invasion: the Placenta Accreta Index. Am J Obstet Gynecol. 2015;212:343.e1-7.

9. Henderson LK, Craig JC, Willis NS, Tovey D, Webster AC. How to write a Cochrane systematic review. Nephrology. 2010;15:617-24.

10. NHS Centre for Reviews and Dissemination. Systematic reviews: CRD's guidance for undertaking reviews in health care. York (UK): University of York, 2009.

11. Leeflang MM, Deeks JJ, Gatsonis C, Bossuyt PM; Cochrane Diagnostic Test Accuracy Working Group. Systematic reviews of diagnostic test accuracy. Ann Intern Med. 2008;149:889-97.
12. Prisma statement. Available on line at: http://www.prismastatement.org/ (accessedMarch 10, 2017).

13. Bossuyt PM, Reitsma JB, Bruns DE, Gatsonis CA, Glasziou PP, Irwig LM, et al. ; Standards for Reporting of Diagnostic Accuracy. Towards complete and accurate reporting of studies of diagnostic accuracy: the STARD initiative. Ann Intern Med. 2003;138:40-4.

14. Benirschke K, Kaufmann P, Baergen RN. Pathology of the human placenta, 5th edn. New York: Springer-Verlag, 2006.

15. Palacios-Jaraquemada JM, Bruno CH, Martín E. MRI in the diagnosis and surgical management of abnormal placentation. Acta Obstet Gynecol Scand. 2013;92:392-7.

16. Collins SL, Ashcroft A, Braun T, Calda P, Langhoff-Roos J, Morel O, et al. ; European Working Group on Abnormally Invasive Placenta (EW-AIP). Proposal for standardized ultrasound descriptors of abnormally invasive placenta (AIP). Ultrasound Obstet Gynecol. 2016;47:271-5.

17. Whiting PF, Rutjes AW, Westwood ME, Mallett S, Deeks JJ, Reitsma JB, et al. ; QUADAS-2 Group. QUADAS-2: a revised tool for the quality assessment of diagnostic accuracy studies. Ann Intern Med. 2011;155:529-36.

18. Rutter CM, Gatsonis CA. A hierarchical regression approach to meta-analysis of diagnostic test accuracy evaluations. Stat Med. 2001;20:2865-84.

19. Glas AS, Lijmer JG, Prins MH, Bonsel GJ, Bossuyt PM. The diagnostic odds ratio: a single indicator of test performance. J Clin Epidemiol. 2003;56:1129-35.

20. Cochrane Handobook for Systematic Reviews of Diagnostic Test Accuracy, Chapter 10. Available online at: http://srdta.cochrane.org/handbook-dta-reviews.

21. Hunter JP, Saratzis A, Sutton AJ, Boucher RH, Sayers $\mathrm{RD}$, Bown MJ. In meta-analyses of proportion studies, funnel plots were found to be an inaccurate method of assessing publication bias. J Clin Epidemiol. 2014;67:897903.

22. Higgins JPT, Green S (editors). Cochrane Handbook for Systematic Reviews of Interventions Version 5.0.2 [updated September 2009]. The Cochrane Collaboration, 2009. Available online at: http://www.cochrane-handbook.org. (accessed March 1, 2017).

23. Egger M, Davey Smith G, Schneider M, Minder C. Bias in meta-analysis detected by a simple, graphical test. BMJ. 1997;315:629-34.

24. Harbord RM, Whiting P. Metandi: meta-analysis of diagnostic accuracy using hierarchical logistic regression. Stata J. 2009;9:211-29.

25. Zamora J, Abraira V, Muriel A, Khan K, Coomarasamy A. Meta-DiSc: a software for meta-analysis of test accuracy data. BMC Med Res Methodol. 2006;6:31.

26. Hashem LB, Salem DS, Hamed ST, Hussein AM. Role of MRI versus ultrasound in the assessment of placental abnormalities and diseases. Egyptian J Radiol Nuclear Med. 2016;47:641-58. 
27. Budorick NE, Figueroa R, Vizcarra M, Shin J. Another look at ultrasound and magnetic resonance imaging for diagnosis of placenta accreta. J Matern Fetal Neonatal Med. 2016;24:1-6.

28. Balcacer P, Pahade J, Spektor M, Staib L, Copel JA, McCarthy S. Magnetic resonance imaging and sonography in the diagnosis of placental invasion. J Ultrasound Med. 2016;35:1445-6.

29. Kumar I, Verma A, Ojha R, Shukla RC, Jain M, Srivastava A. Invasive placental disorders: a prospective US and MRI comparative analysis. Acta Radiol. 2017;58:121-8.

30. Aitken K, Allen L, Pantazi S, Kingdom J, Keating S, Pollard L, et al. MRI Significantly improves disease staging to direct surgical planning for abnormal invasive placentation: a single centre experience. J Obstet Gynaecol Can. 2016;38:246-251.e1.

31. Algebally AM, Yousef RR, Badr SS, Al Obeidly A, Szmigielski W, Al Ibrahim AA. The value of ultrasound and magnetic resonance imaging in diagnostics and prediction of morbidity in cases of placenta previa with abnormal placentation. Pol J Radiol. 2014;79:409-16.

32. Chalubinski KM, Pils S, Klein K, Seemann R, Speiser P, Langer $\mathrm{M}$, et al. Prenatal sonography can predict degree of placental invasion. Ultrasound Obstet Gynecol. 2013;42:518-24.

33. Peker N, Turan V, Ergenoglu M, Yeniel O, Sever A, Kazandi M, et al. Assessment of total placenta previa by magnetic resonance imaging and ultrasonography to detect placenta accreta and its variants. Ginekol Pol. 2013;84:18692.

34. Calì G, Giambanco L, Puccio G, Forlani F. Morbidly adherent placenta: evaluation of ultrasound diagnostic criteria and differentiation of placenta accreta from percreta. Ultrasound Obstet Gynecol. 2013;41:406-12.

35. Gohuar GK, Sadek SM, Siam S, Ahmad RA. Role of transperineal sonography in diagnosis of placenta previa/ accreta: a prospective study. Egyptian J Radiol Nuclear Med. 2012;43:637-45.

36. Woodring TC, Klauser CK, Bofill JA, Martin RW, Morrison JC. Prediction of placenta accreta by ultrasonography and color doppler imaging. J Matern Fetal Neonatal Med. 2011;24:118-21.

37. Lim PS, Greenberg M, Edelson MI, Bell KA, Edmonds PR, Mackey AM. Utility of ultrasound and MRI in prenatal diagnosis of placenta accreta: a pilot study. AJR Am J Roentgenol. 2011;197:1506-13.

38. Chou MM, Chen WC, Tseng JJ, Chen YF, Yeh TT, Ho ES. Prenatal detection of bladder wall involvement in invasive placentation with sequential two-dimensional and adjunctive three-dimensional ultrasonography. Taiwan J Obstet Gynecol. 2009;48:38-45.

39. Masselli G, Brunelli R, Casciani E, Polettini E, Piccioni $\mathrm{MG}$, Anceschi $\mathrm{M}$, et al. Magnetic resonance imaging in the evaluation of placental adhesive disorders: correlation with color Doppler ultrasound. Eur Radiol. 2008;18:1292-9.

40. Miura K, Miura S, Yamasaki K, Yoshida A, Yoshiura K, Nakayama D, et al. Increased level of cell-free placental mRNA in a subgroup of placenta previa that needs hysterectomy. Prenat Diagn. 2008;28:805-59.

41. Japaraj RP, Mimin TS, Mukudan K. Antenatal diagnosis of placenta previa accreta in patients with previous cesarean scar. J Obstet Gynaecol Res. 2007;33:431-7.

42. Yang JI, Lim YK, Kim HS, Chang KH, Lee JP, Ryu HS. Sonographic findings of placental lacunae and the prediction of adherent placenta in women with placenta previa totalis and prior Cesarean section. Ultrasound Obstet Gynecol. 2006;28:178-82.

43. Comstock CH, Love JJ Jr, Bronsteen RA, Lee W, Vettraino IM, Huang RR, et al. Sonographic detection of placenta accreta in the second and third trimesters of pregnancy. Am J Obstet Gynecol. 2004;190:1135-40.

44. Twickler DM, Lucas MJ, Balis AB, Santos-Ramos R, Martin L, Malone S, et al. Color flow mapping for myometrial invasion in women with a prior cesarean delivery. J Matern Fetal Med. 2000;9:330-5.

45. Chou MM, Ho ES, Lee YH. Prenatal diagnosis of placenta previa accreta by transabdominal color Doppler ultrasound. Ultrasound Obstet Gynecol. 2000;15:28-35.

\section{Supporting information}

Additional Supporting Information may be found in the online version of this article:

Table S1. Search strategy.

Table S2. Excluded studies and reason for the exclusion.

Table S3. Results of the meta-analyses evaluating the association between selected ultrasound signs and degrees of placental invasion.

Table S4. Pooled rates of: (a) placenta accreta; (b) placenta increta; (c) placenta accreta+increta; (d) placenta percreta in pregnancies with (left) and without (right) each ultrasound sign. Data from single studies have been combined using proportion meta-analysis (random-effect model). For each outcome, the pooled rates were computed only when more than one study could be included in the corresponding meta-analysis. 